\title{
Applications of Mathematical Theorems and laws in Electrical Engineering (Kirchhoff's Laws)
}

\author{
Daw Nan Ei Ei Theint \\ Lecturer \\ Departments of Engineering Mathematics, \\ Technological Universities (Toungoo) \\ Toungoo ,Myanmar
}

\author{
Dr. Soe Soe \\ Professor and Head \\ Departments of Engineering Mathematics, \\ Technological Universities (Sagaing) \\ Sagaing ,Myanmar
}

\begin{abstract}
In this paper, not only the essential concepts are known but also the Kirchhoff's laws are derived by the applications of Mathematical Theorem and laws, Divergence Theorem, Gauss's law. Moreover, laws, that is a relation between electric field and magnetism, Faraday's law of induction and Ampere's law, is applied to be obtained Kirchhoff's laws. Then, the electrical engineering problems concerned with Kirchhoff's laws will be solved
\end{abstract}

Keywords: Gauss, Faraday, Ampere, Kirchhoff, electric field, magnetic field, total electric current density, charge density.

\section{INTRODUCTION}

We have known that "a network is the interconnection of two or more simple devices" and "a circuit is a network which contains at least one closed path" Thus, every circuit is a network but not all networks are circuits. In electric circuit analysis, it is important to be known the concepts and conventions, the unit and quantities used in circuit analysis, used in introductory circuit analysis. Several practical examples to be illustrated these concepts. [1]

Now in this paper, firstly the conventions required for this paper and the basic concepts, electric current, ampere, voltage (potential difference), power, and sources of energy, i.e., ideal independent sources and dependent sources, active and passive devices, circuits and networks, active and passive networks are introduced. Secondly, international system of units, necessary conditions for current flow will be interpreted. Then, Ohm's law, the definitions of Nodes, Branches, Loops and Meshes will be illustrated.

2. Basic Concepts of Electric current, Ampere, Voltage, Power, Ideal Independent Sources and Dependent Sources, Active and Passive Devices, Circuits and Networks, Active and Passive Networks

\subsection{Conventions}

In this paper, the coulomb, the instantaneous values of voltage, current and power are represented by $q, v, i$ and $p$ respectively. Subscripts will be used to denote specific voltages, currents, resistances, etc. For example, vA or v1 will represent the voltage(potential difference) between point $\mathrm{A}$ or point 1 with respect to some arbitrarily chosen reference point taken as "zero" volts or "ground".

\subsection{The Coulomb}

The coulomb, abbreviated as $\mathrm{C}$, is the fundamental unit of electric charge. In terms of this unit, the charge of an electron is $-1.6 \times 10^{-19} \mathrm{C}$. We have also known that one negative coulomb is equal to $6.24 \times 10^{18}$ electrons.

\subsection{Electric Current and Ampere}

Electric current, $i$, at a specified point and flowing in a specified direction is defined as the instantaneous rate at which net positive charge is moving past this point in that specified direction.

In symbol,

$$
i=\frac{d q}{d t}=\lim _{\Delta t \rightarrow 0} \frac{\Delta q}{\Delta t} .
$$

The unit of current is the ampere abbreviated as A and corresponds to charge $q$ moving at the rate of one coulomb per second. In other words,

$$
1 \text { ampere }=\frac{1 \text { coulomb }}{1 \text { second }}
$$

\subsection{Voltage (Potential Difference)}

The voltage (potential difference) across a two-terminal device is defined as the work required to move a positive charge of one coulomb from one terminal of the device to the other terminal.

$$
1 \text { volt }=\frac{1 \text { joule }}{1 \text { coulomb }} \text {. }
$$

\subsection{Ideal Independent Sources and Dependent Sources}

Ideal Independent Voltage Source may maintain same voltage regardless of the amount of current that flows through it. Its value is either constant (DC) or sinusoidal (AC).

Ideal Independent Current Source may maintain same current regardless of the voltage that appears across its terminals. Its value is either constant (DC) or sinusoidal (AC).

Dependent Voltage Source -Its value depends on another voltage or current elsewhere in the circuit. Here, $\mathrm{k}_{1}$ is a constant and $\mathrm{k}_{2}$ is a resistance as defined in linear devices below. When denoted as $\mathrm{k}_{1} \mathrm{v}$ it is referred to as voltage controlled voltage source, and when denoted as $\mathrm{k}_{2} i$ it is referred to as current controlled voltage source.

Dependent Current Source -Its value depends on another current or voltage elsewhere in the circuit. Here, $\mathrm{k}_{3}$ is a constant and $\mathrm{k}_{4}$ is a conductance as defined in linear devices below. When denoted as $\mathrm{k}_{3} i$ it is referred to as current 
controlled current source and when denoted as $\mathrm{k}_{4} \mathrm{~V}$ it is referred to as voltage controlled current source.

\subsection{Circuits and Networks}

A network is the interconnection of two or more simple devices.

A circuit is a network which contains at least one closed path. Thus every circuit is a network but not all networks are circuits.

\section{Necessary Conditions for Current flow 3.1 Necessary Conditions for Current Flow}

There are two conditions which are necessary to set up and maintain a flow of current in a network or circuit. These are:

(a) There must be a voltage source (potential difference) present to provide the electrical work which will force current to flow.

(b) The circuit must be closed.

\subsection{Ohm's Law}

Ohm's law states that the voltage or potential difference between two points is directly proportional to the current or electricity passing through the resistance, while the current or electricity passing through the resistance is inversely proportional to the resistance of the circuit. The formula for Ohm's law is $v_{R}=i_{R} R$.

This relationship between current and voltage was discovered by German scientist Georg Simon Ohm.

\subsection{Node of Electric Circuit}

The point through which an electric circuit element is connected to the circuit is called node. It is better to say, node is a point where, terminal of two or more circuit elements are connected together. Node is a junction point in the circuit.

\subsection{Loops in Electric Circuit}

An electric circuit has numbers of nodes. If one starts from one node and after going through a set of nodes returns to same starting node without crossing any of the intermediate node twice, i.e., loop is any closed path in the circuit formed by branches.

\subsection{Mesh in Electric Circuit}

A mesh is a closed path in a circuit with no other paths inside it. In other words, a match is a loop with no other loops inside it.

\section{Kirchhoff's Law}

In this section, Kirchhoff's laws may be obtained by the applications of Mathematical Theorems and laws. Moreover, the laws concerned with Physics and Chemistry, i.e. Ohm's law and Faraday's law may also be applied.

\subsection{Kirchhoff's Current Law (KCL)}

Kirchhoff's Current Law states that "The algebraic sum of currents in a network of conductors meeting at a point is zero."

Proof:

Kirchhoff's Current Law is obtained by being used of divergence theorem, Gauss's law, ampere law and Clairaut's Theorem.
We now consider a point in a network of conductors with $\mathrm{N}$ number of wires emanating from it. Enclose the node with some open bounded nonempty region, $\Omega \subseteq R^{2}$, with boundary $\mathrm{S}$. We denoted by $\mathrm{S}_{\mathrm{k}}$ the intersection of the $\mathrm{k}^{\text {th }}$ wire and the surface $\mathrm{S}$.

It an electric current is applied in this circuit. Then, electric current density $(J)$ is derived with an application of divergence theorem is used to be solved both volume and surface integral

$$
\iiint_{\Omega} \nabla \cdot J d V=\iint_{S} J \cdot \hat{n} d S
$$

Left hand side of Equation (1) is expressed for volume of current inside the network and it is derived by volume integral but at one node, it is consider only for current area, so surface integral is used. Now, the surface integral contains two portions, one is for the surface integral for the number of $\mathrm{k}$ wires in the considered domain and the last is considered outside this domain. Thus, Equation (1) may be written as

$\iiint_{\Omega} \nabla \cdot J d V=\sum_{k=1}^{N} \iint_{S} J \cdot \hat{n} d S+\iint_{S}\left(u_{k=1}^{N} s_{k} J \cdot \hat{n} d S\right.$ (2)

The second term in the left hand side is zero because the current density $\mathbf{J}$ outside the domain is zero. Thus, equation (2) becomes

$$
\iiint_{\Omega} \nabla \cdot J d V=\sum_{k=1}^{N} \iint_{S} J \cdot \hat{n} d S(3)
$$

Left-hand side of Equation (3) is the total sum of current in this domain. Because $\iint_{S} J \cdot \hat{n} d S$ is the dot product of density of the current flow $\mathrm{J}$ and the volume $\iint_{S} n d S$ and the currents flowing in each wire for $k=1,2,3, \ldots, N$ is obtained and then, their current sum may be obtained by Equation (4)

$$
\iiint_{\Omega} \nabla \cdot J d V=\sum_{k=1}^{W} i_{k}(4)
$$

where, the current $\hat{t}_{\mathbb{k}}$ is signed, with negative current indicating that the current is going inside the point. $\iiint_{\Omega} \nabla \cdot J d V$ may be started with Ampere's law, it is a relation between electric field and magnetic field.Then

$$
\begin{aligned}
& \nabla \times \vec{B}=\mu\left(\epsilon \frac{\partial \vec{E}}{\partial t}+J\right)(5) \\
& \frac{\partial \vec{D}}{\partial t}+J=\nabla \times \vec{H}(6)
\end{aligned}
$$

where $\mathrm{D}$, the flux of electric field density, is equal to $E E$ and $\mathrm{H}$, the flux of magnetic field, and it is equal to $\frac{\beta}{\mu}$.

Being applied the gradient operator on both sides of equation (6), we get

$$
\nabla \cdot \frac{\partial \vec{D}}{\partial t}+\nabla_{x} J=\nabla_{\cdot}(\nabla \times \vec{H}) .(7)
$$

We have $\nabla \cdot(\nabla \times \vec{H})=0$, equation (7) becomes

$$
\nabla \cdot \frac{\partial \vec{D}}{\partial t}+\nabla_{x} J=0 \rightarrow \nabla_{x} J=-\nabla_{x} \frac{\partial \vec{D}}{\partial t} \cdot(8)
$$

Since the intersection of the $\mathrm{k}^{\text {th }}$ wire and the surface $\mathrm{S}$ is considered, Clairaut's Theorem is applied to switch the order of derivatives, right side of Equation (8) may be written as

$$
\nabla \cdot \frac{\partial \vec{D}}{\partial t}=\frac{\partial}{\partial t}(\nabla \cdot \vec{D})(9)
$$

From Equations (9), the divergence of electric flux may give the charge density, $\rho$ and this phenomenon is known by Gauss's law. Equation (8) may be written as 


$$
\nabla \cdot J=-\frac{\partial}{\partial t}(\nabla \cdot \vec{D})=-\frac{\partial \rho}{\partial t}(10)
$$

Being taken integrating over the region $\Omega$, we get

$$
\iiint_{\Omega} \nabla \cdot J d V=\iiint_{\Omega}-\frac{\partial \rho}{\partial t} d V(11)
$$

If the charge density $\rho$ and $\frac{\partial \rho}{\partial t}$ are considered continuous function, $\frac{\partial}{\partial t}$ can be taken outside the integral, then equation (11) may be written as

$$
\begin{aligned}
& \iiint_{\Omega} \nabla \cdot J d V=-\frac{d}{d t} \iiint_{\Omega} \rho d V \times(12) \\
& \iiint_{\Omega} \nabla_{x} J d V \text { is the integrand of the }
\end{aligned}
$$
product of charge density and its volume so we get the total charge $\mathrm{Q}(\mathrm{t})$ is enclosed in the region $\Omega$ at time $\mathrm{t}$,

$$
\iiint_{\Omega} \rho d V=Q(t) .(13)
$$

Thus, equation (12) becomes

$$
\iiint_{\Omega} \nabla \cdot J d V=-\frac{d}{d t} Q(t) .(14)
$$

If the charge $Q(t)$ is conserved, i.e., $Q(t)=Q$, where $Q$ is a constant with respect to $t$, then $\frac{d}{d t} Q(t)=0$.

The above equation becomes

$$
\iiint_{\Omega} \nabla . J d V=0 .(15)
$$

Then, right hand side of equation (4) becomes

$$
\sum_{k=1}^{N} i_{k}=0 .(16)
$$

Equation (16) satisfies the Kirchhoff's current Law.

\subsection{Kirchhoff's Voltage Law (KVL)}

Kirchhoff"s Voltage Law states that "The algebraic sum of all the voltages around any closed path in a circuit is zero."

Proof:

Kirchhoff's voltage Law is obtained by being used of Faraday's Law and Stokes' theorem.

Kirchhoff's voltage Law is applied DC stationary circuits, with magnetic field density constant with respect to time. If a closed electrical circuit with along a path $\mathrm{C}$ is considered and $\mathrm{S}$ is assumed any surface with the boundary C.

Faraday's Law is defined as

$$
\nabla \times \vec{E}=-\frac{\partial \overrightarrow{\vec{E}}}{\partial t}(1)
$$

By being integrated the above equation on the surface $S$, equation (1) becomes

$$
\iint_{S}(\nabla \times \vec{E}) \cdot \hat{n} d S=-\iint_{S} \frac{\partial \vec{B}}{\partial t} \cdot \hat{n} d S
$$

Left hand side of equation (2) is applied to Stokes' theorem, then

$$
\iint_{S}(\nabla \times \vec{E}) \cdot \hat{n} d S=\oint_{C} \vec{E} \cdot d \vec{r}(3)
$$

So, right hand sides of equation (2) and equation (3) becomes equal.

$$
\oint_{C} \vec{E} \cdot d \vec{r}=-\iint_{S} \frac{\partial \vec{B}}{\partial t} . \hat{n} d S(4)
$$

Since the electrical circuit is closed, $\left(n_{k}\right)_{k=0}^{N}$ with $n_{0}=n_{N}$ be any ordered points, for any parameterization $\vec{r}:[a, b] \rightarrow C$, with $r\left(t_{k}\right)=n_{k}$, we have $t_{k}<t_{k+1}$, for $\mathrm{k}$ is from zero to $\mathrm{N}-1$.

$$
\text { Thus, } r\left(t_{0}=a\right)=r\left(t_{N}=b\right)=n_{0}=n_{N} \text {. }
$$

$$
\oint_{C} \vec{E} \cdot d \vec{r}=\sum_{k=1}^{W} \oint_{C_{k}} \vec{E} \times d \vec{r}(5)
$$

where, $C_{k}$ denotes the segment of the curve $\mathrm{C}$ between the point $n_{k-1}$ and $n_{k}$.

We have the definition

$$
\oint_{C_{k}} \vec{E} \cdot d \vec{r}=V_{k}(6)
$$

where, $V_{k}$ is the voltage between the points $n_{k-1}$ and $n_{k}$.

By being used equations (4), (5) and (6), we get

$$
\sum_{k=1}^{N} V_{k}=-\iint_{S} \frac{\partial \vec{B}}{\partial t} . \hat{n} d S(7)
$$

If the magnetic field density is constant with respect to time, then

$$
\frac{\partial \vec{B}}{\partial t}=0
$$

Thus equation (7) becomes

$$
\sum_{k=1}^{N} V_{k}=0 \text { (9) }
$$

The last equation shows that Kirchhoff's Voltage Law is satisfied.

\subsection{Applications of Kirchhoff's Laws}

Problem (1)We consider an analysis of a circuit to solve a problem containing dependent sources shown in the following circuit diagram.

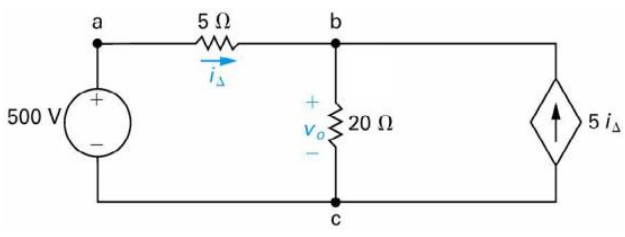

Solution

Figure (1)

Ohm's law and Kirchhoff's Laws are applied to find $v_{0}$.

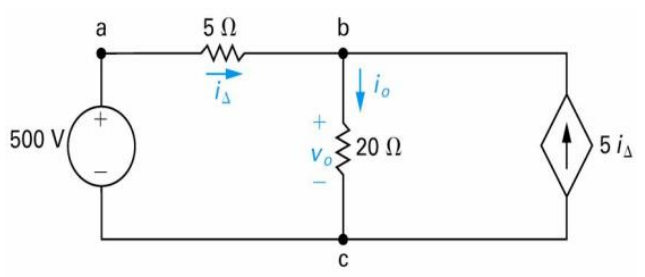

Figure (2)

Let io be the current flowing on the $20 \Omega$ resistor. By being applied Ohm's law, $\mathrm{v}_{0}=\mathrm{i}_{0} 20(1)$

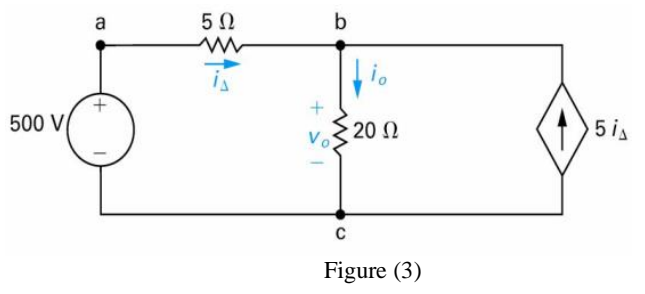

KCL will provide us with one equation relating io and $\mathrm{i}_{\Delta}$ namely.

$$
\begin{aligned}
& \text { At Node b } \\
& i_{\Delta}+5 i_{\Delta}-i_{0}=0 \rightarrow 6 i_{\Delta}-i_{0}=0 .
\end{aligned}
$$


(4), (5) and (6).

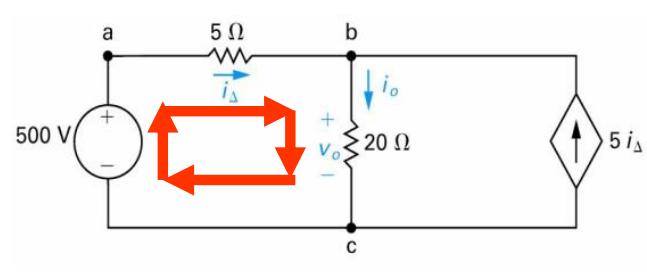

Figure (4)

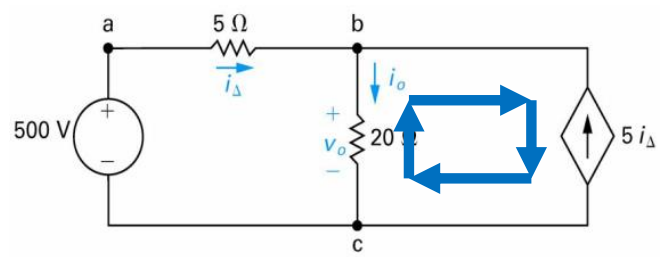

Figure (5)

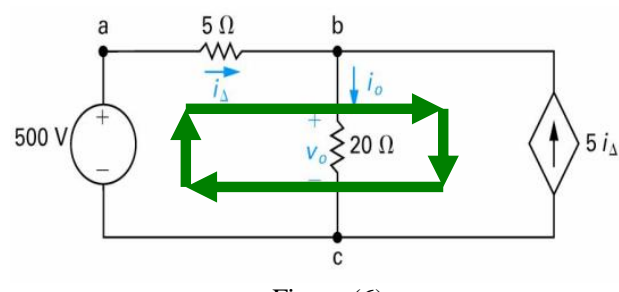

Figure (6)

Only the loop abca shown in Figure (6) may be applied KVL because this loop does not contain a current source and the other two loops contain a current source, so KVL may not be applied to those loops.

By being applied KVL to the loop abca, we get,

$-500+5 i_{\triangle}+20 i_{0}=0 \rightarrow 5 i_{\triangle}+20 i_{0}=500(3)$

By being solved equations (2) and (3), we obtain

$i_{0}=24 \mathrm{~A} \rightarrow v_{0}=480 \mathrm{~V}$ and $i_{\Delta}=4 \mathrm{~A}$.

(https://www.toppr.com )(REFERENCE-8)

\section{Conclusion}

In my conclusion, to be proved Kirchhoff's Laws, we must know not only the Mathematical concepts, i.e., Gauss Law and Divergence Theorem but also the laws concerned with Physics, i.e., Faraday's Law and Ohm's Law. Moreover, to be solved the Electrical Engineering problems with Kirchhoff's Laws, the essential concepts, loops in electric circuits, node of electric circuit, etc., may be known. So all of these concepts must be known to solve an Electrical Engineering problems with the applications of Kirchhoff's Laws.

\section{ACKNOWLEDGEMENT}

Firstly, I would like to give my acknowledgement to Dr. Soe Soe, Professor \& Head of Engineering Mathematics Department, Technological University (Sagaing), as her encourage for this paper together with me and advised me to perform this paper the best. Secondly to Rector, Technological University(Toungoo) and Dr. Yu Yu Khin,
Head of Engineering Mathematics Department, TU(Toungoo), as she let me go to TU (Sagaing) to perform this paper. Finally, I am also grateful to all of the members who accepted my paper to be published in this journal.

\section{REFERENCES}

[1] Steven, T.Karris, Circuit Analysis I with MATLAB Applications, Orchard Publications, Fremont, California.www.orchardpublications.com.

[2] Giorgio Rizzoni, Fundamentals of Electrical Engineering, $1^{\text {st }}$ Edition, 2008.

[3] By Tony R. Kuphaldt, Lessons In Elcctric Circuits Volume II-AC, Sixth Edition, Last up-date July 25,2007.

[4] Francis, Bacon, Fundamentals of Electric Circuits.

[5] Free Online Course Materials.Physics.MIT. Open Course Ware. "Web.28 Feb.2012".

[6] Hans C. Ohanian, John T. Markert.28 Feb.2012.

[7] Prashant Athavale, "Kirchhoff's current Law and Kirchhoff's voltage Law".

[8] https://www.toppr.com. 